Meta

Journal des traducteurs

Translators' Journal

\title{
La contribution de la terminologie à la conception théorique des langages documentaires et à l'indexation de documents
}

\section{Widad Mustafa-Elhadi}

Volume 37, numéro 3, septembre 1992

URI : https://id.erudit.org/iderudit/002699ar

DOI : https://doi.org/10.7202/002699ar

Aller au sommaire du numéro

Éditeur(s)

Les Presses de l'Université de Montréal

ISSN

0026-0452 (imprimé)

1492-1421 (numérique)

Découvrir la revue

Citer cet article

Mustafa-Elhadi, W. (1992). La contribution de la terminologie à la conception théorique des langages documentaires et à l'indexation de documents. Meta, 37(3), 465-473. https://doi.org/10.7202/002699ar
Résumé de l'article

This paper attempts to show the contribution of certain linguistic phenomena, and reciprocally, the contribution of linguistics to the analysis of semantic relationships used by the documentalists in conceiving thesauri. Abstract representation of extra-linguistic reality involves understanding of certain designation processes which are useful for linguistic research. Taking into account these elements would help achieving a better collaboration between linguists, documentalists and information systems designers. 


\title{
LA CONTRIBUTION \\ DE LA TERMINOLOGIE \\ À LA CONCEPTION THÉORIQUE DES LANGAGES DOCUMENTAIRES ET A L'INDEXATION DE DOCUMENTS
}

\author{
WIDAD MUSTAFA-ELHADI \\ Université Stendhal Grenoble 3, CRTT, \\ Université Lumière Lyon 2, Lyon, France
}

\begin{abstract}
This paper attempts to show the contribution of certain linguistic phenomena, and reciprocally, the contribution of linguistics to the analysis of semantic relationships used by the documentalists in conceiving thesauri. Abstract representation of extra-linguistic reality involves understanding of certain designation processes which are useful for linguistic research. Taking into account these elements would help achieving a better collaboration between linguists, documentalists and information systems designers.
\end{abstract}

\section{INTRODUCTION}

La terminologie est un outil de traduction et un outil d'indexation. De ce fait, elle occupe une place prépondérante dans les sciences de l'information et de la communication. Mais la spécificité de cette discipline est-elle évidente? Dans le meilleur des cas, elle est considérée comme une discipline connexe de la lexicologie ou comme faisant partie du lexique; dans le pire des cas, elle est assimilée au processus de la traduction.

Sa séparation d'avec la lexicologie et la traduction n'est pas une révélation: on trouve les traces de cette séparation dans la réflexion philosophique sur le langage, notamment chez les logiciens de Port-Royal et leurs successeurs, John Locke, philosophe anglais du XVII ${ }^{\mathrm{e}}$ siècle, et bien d'autres dont la pensée a perpétué cette tradition.

Ce courant se concrétise à la fin du $\mathrm{XIX}^{\mathrm{e}}$ siècle et au début du $\mathrm{XX}^{\mathrm{e}}$ siècle, et les outils de description deviennent de plus en plus précis. Si John Locke avait eu l'intuition de la nature du terme comme nom de classe, Charles Sanders Peirce l'a, quant à lui, indiqué clairement dans le cadre de sa sémiotique. Les travaux de Gottlob Frege sur le concept, le sens et la référence ont constitué un tournant dans la recherche fondamentale en terminologie.

Le schéma de classification est un essai de la transposition de la réalité concrète ou abstraite. Mais la réalité est souvent appréhendée selon divers angles, d'où l'idée des classifications hiérarchiques et à facettes ou points de vue, idée clairement formulée par les langages documentaires ${ }^{1}$ très en vogue au $\mathrm{XX}^{\mathrm{e}}$ siècle : les langages à structure hiérarchique et ceux à structure combinatoire ${ }^{2}$. Il est à signaler que la conception et le développement de thésaurus ${ }^{3}$ a joué un rôle important dans le perfectionnement de langages documentaires et, par conséquent, dans le développement de la recherche en terminologie.

La réalité ainsi «décrite» est nommée par la langue qui est organisée en un double système de nomination et de communication. On classe les concepts représentant des objets; cependant, chaque concept est désigné par un terme. De ce fait, on se trouve 
devant une relation triadique : le terme est une unité linguistique désignant un ensemble de propriétés ou concept représentant un objet dans un référentiel déterminé. La séparation entre les trois niveaux s'impose comme une exigence à la fois théorique et pratique.

La terminologie d'une langue donnée recrute les unités terminologiques parmi les lexèmes de cette langue. Le sens de chacun de ses lexèmes peut être appréhendé indépendamment de la prise en compte d'un univers donné. Le lexème recruté par la terminologie est, lui, nécessairement pris dans un univers donné qu'on appelle communément domaine. Le lexème peut ne pas renvoyer à un référent. Le terme, lui, renvoie nécessairement à un référent, d'où la distinction obligée entre le sens et la référence.

\section{LES POSSIBILITÉS OFFERTES PAR LE SYSTÈME DE NOMINATION}

Pour donner des étiquettes aux classes d'objets «l'Homme a eu recours à une combinatoire 4 linguistique. Cette combinatoire devait produire ses langues comme autant de 'systèmes de systèmes', des systèmes composés chacun de quatre systèmes interdépendants: un système de phonèmes, un système de syllabes, un système de nomination, un système de communication ${ }^{5}$ ».

L'opération de nomination est la mise en relation de deux entités: une entité linguistique et une entité extra-linguistique. Cette opération révèle une classification dans la mesure où une étiquette comme «cheval» désigne toute la classe des chevaux. D'où la considération du terme comme nom de classe. Un système de nomination qui produirait une seule forme pour une seule unité référentielle serait un système idéal. En réalité, un tel système épuiserait très rapidement ses ressources, car les combinaisons possibles sont limitées.

Le processus de dénomination suit mal, quelle que soit la langue, le rythme accéléré de «production» de concepts dans les diverses disciplines de l'activité humaine. La demande en termes peut dépasser la capacité d'invention et de mémorisation des utilisateurs.

L'essor terminologique se traduit par le besoin pressant de termes pour nommer des réalités nouvelles en nombre sans cesse croissant. Or une langue ne saurait toujours suivre ce rythme sans réemployer son stock de mots malgré le recours aux syntagmes nominaux.

Parmi les moyens dont les langues disposent pour réemployer leurs unités pour nommer les classes d'objets nouveaux, il y a les tropes: la métaphore et la métonymie. Et s'il y a réemploi d'une unité, il y a par conséquent polysémie, caractéristique peu désirable dans les domaines de la terminologie et de la documentation. Cependant, l'examen des définitions des concepts ainsi que la classification à facettes du domaine des télécommunications montrent qu'il s'agit là d'une polysémie d'un type particulier, comme nous le verrons dans la troisième partie de cet article.

\section{LES POSSIBILITÉS OFFERTES PAR LE SYSTÈME DE COMMUNICATION}

«Le système de communication d'une langue ou syntaxe est l'organisation des unités de nomination de cette langue en un système de relations qui structure et fait une certaine unité de communication : la phrase ${ }^{6} \gg$. Parler de la syntaxe dans ce cadre peut paraître de prime abord non pertinent. Mais si l'on examine un recueil de termes, le nombre de syntagmes nominaux, parfois très longs, justifiera la place de la syntaxe dans une terminologie. Ces longs syntagmes révèlent l'impossibilité pour une langue de dépendre de son seul système de nomination malgré les extensions du sens par les tropes que nous examinerons plus loin. Si l'on veut, par exemple, nommer les sous-classes de réseaux appel, signal, circuit, faisceau, transmission, etc. - , le recours au système de communication s'avère obligatoire. Sur les propriétés de l'objet à nommer, le système de commu- 
nication opère un codage qui permet diverses combinaisons d'unités linguistiques. Prenons par exemple la classe des circuits :

circuit $=\Rightarrow$ circuit électronique, circuit électromagnétique, circuit loué, circuit logique, etc.

D'autre part, ces combinaisons révèlent les classifications fines (sous-classes de classes) sous-jacentes au processus de nomination, qui facilitent l'établissement de relations de type sémantique: relations hiérarchiques (générique/spécifique, partitive), d'équivalence ou synonymiques, polysémiques. Ces deux dernières sont particulièrement dues aux termes issus d'hypallages ${ }^{7}$, qui naissent des réductions de syntagmes nominaux longs: réseau de synchronisation despotique $===>$ réseau despotique (équivalence); réseau à synchronisation mutuelle hiérarchique $===>$ réseau hiérarchique (cas limite de polysémie qui peut même être considéré comme un cas d'homonymie, car réseau hiérarchique désigne aussi les réseaux d'ordinateurs qui sont une sous-classe des réseaux locaux d'informatique).

\section{LES LANGAGES DOCUMENTAIRES ET LEUR RÔLE DANS UNE RECHERCHE TERMINOLOGIQUE}

Une terminologie fiable exige des concepts exactement définis et une bonne structuration conceptuelle du domaine étudié. Cette structuration, sous forme d'un schéma arborescent, communément appelé «arbre du domaine», sert à décomposer ce domaine afin d'identifier ses principales parties constitutives ou sous-domaines, lesquels seront aussi décomposés en sous-domaines. Autrement dit, le domaine sera constitué de classes de classes, conformément à la logique des classes de Port-Royal. L'arbre du domaine, de même que les autres représentations d'un domaine, est représenté en terminologie par une langue naturelle pour des raisons de commodité.

Les structures de représentation sont empruntées aux langages documentaires et classificatoires, ce qui nous amène à montrer ce qui les rattache à la terminologie et ce qui les différencie.

Élément commun aux deux disciplines, — la TERMINOLOGIE et la DOCUMENTATION -, le terme est, d'une part, l'unité linguistique qui sert à désigner un concept appartenant à une discipline de l'activité humaine et, d'autre part, l'unité qui sert à l'indexation des documents dans les systèmes d'information et de documentation. Quand il sert à l'indexation des documents, il est alors appelé communément descripteur, le terme ne pouvant ici être autre chose que le terme préférentiel que le documentaliste choisira parmi tous les termes qui se trouvent être autant de candidats-descripteurs.

$\mathrm{Si}$ les langages documentaires servent, à travers la classification, au classement physique des documents dans les bibliothèques, rôle pour lequel ils ont été conçus au départ, leur emploi s'est étendu à la classification des domaines de l'activité humaine. Cependant, ces deux rôles portent un seul nom: l'«organisation du savoir universel».

Le recours à plusieurs types de langages documentaires lors de la structuration du domaine des télécommunications - les langages à structure hiérarchique, les langages à structure combinatoire ou à structure multidimensionnelle, la classification à facettes ou «Colon Classification» et les «Tableaux de l'Organum ${ }^{{ }}$» de l'Encyclopcedia Universalis - nous a permis d'examiner de près les avantages que présente l'intégration des méthodes de classification utilisées par les concepteurs et théoriciens de la documentation dans une recherche terminologique. Certains spécialistes de la documentation reconnaîtront sans doute nos classifications comme des classifications $\left\langle\right.$ hybrides ${ }^{9}$, eux qui distinguent les trois types suivants : 
- les classifications de type monohiérarchique;

- les classifications à facettes;

- les classifications hybrides ${ }^{10}$.

Nombre de documentalistes admettent l'intérêt du type de classification «hybride». Chaque classification documentaire présente en effet des avantages qui lui sont propres, ce qui rend ces types complémentaires.

Ces nouveaux types de classification - à facettes, hybrides, tableaux de l'Organum etc. - offrent davantage de possibilités d'analyse en profondeur de l'ensemble des concepts d'une terminologie. Les langages à structure hiérarchique apparaissent en comparaison «rigides». C'est précisément pour éviter cette «rigidité» qu'ont été construits les «Tableaux de l'Organum», dont les auteurs sont allés jusqu'à mettre en question les classifications hiérarchiques marquées par la logique classique. Le type de relations qu'ils ont adopté se révèle avantageux surtout pour la mise en évidence des relations associatives. Car l'utilisateur d'un thésaurus a le plus grand besoin de ce type de relations qui peuvent faciliter ses recherches du fait qu'elles offrent plusieurs parcours possibles du concept. De ce fait, elles sont pertinentes lors de l'interrogation.

Le thésaurus est considéré par les spécialistes de la documentation comme une représentation symbolique ne prenant pas en compte le référent. Mais le descripteur renvoie à des documents qui traitent de la réalité; cela revient à dire que le descripteur renvoie à cette même réalité par référent interposé, c'est-à-dire par le document.

Dans les terminologies spécialisées, les termes sont accompagnés de leurs définitions, alors que les descripteurs d'un thésaurus sont accompagnés de non-descripteurs le descripteur étant le terme préférentiel et les non-descripteurs étant l'ensemble de ses synonymes - et de relations hiérarchiques, partitives, associatives.

Il serait utile de rapprocher les structures de la terminologie, fondées, elles, sur le référent et la définition, et les structures du thésaurus, lesquelles sont fondées, entre autres, sur les relations sémantiques.

Aussi serait-il intéressant de proposer un répertoire qui retiendrait de la terminologie ses protocoles rigoureux de définition et du thésaurus ses structures variées.

\section{INCIDENCES DE LA RECHERCHE TERMINOLOGIQUE SUR LES LANGAGES DOCUMENTAIRES}

Dans cette partie, nous tâcherons de montrer que terminologues et documentalistes ont intérêt à travailler de concert tout en gardant la spécificité de leurs disciplines respectives. Il s'agit de mettre en évidence l'apport des langages documentaires à l'analyse de certains faits linguistiques et, inversement, ce que la linguistique peut apporter à l'analyse des relations sémantiques utilisées par les documentalistes-concepteurs de thésaurus.

La classification à facettes, dont l'application est directement liée aux insuffisances des classifications monohiérarchiques - rigidité, lourdeur, relative pauvreté des relations sémantiques ${ }^{11}$ - et dont la structure permet la représentation abstraite sous divers angles de la réalité extra-linguistique, a été particulièrement féconde pour l'analyse de certains concepts. Nous avons choisi comme exemples les signaux, réseaux, circuits, faisceaux du domaine des télécommunications. Nous les avons classés dans des arbres à facettes multiples (chaque arbre permet un parcours différent des concepts étudiés). Les arbres sont accompagnés de tableaux précisant la localisation des concepts dans chaque facette. Il est ainsi possible de trouver la classification optimale d'un corpus. De plus, dans l'optique d'une terminologie multilingue, la recherche des équivalences est facilitée.

L'importance de la classification des concepts rigoureusement définis, sans lesquels une terminologie ne peut pas s'établir, révèle d'autres aspects intéressants pour la 
recherche en linguistique. Pour un linguiste-terminologue, la classification ne se limite pas à l'organisation des concepts ni à la représentation abstraite sous divers angles de la réalité extra-linguistique. Elle aide aussi à comprendre certains processus de dénomination. Les combinatoires linguistiques sont révélées par les diverses représentations du réel. Autrement dit, il y a une interaction entre la représentation abstraite du réel et certains mécanismes linguistiques dont le plus important est la métonymie, ce trope ô combien subtil, qui se construit sur le réel et par le réel. Ce trope abonde dans la langue technique. L'usage le plus évident en est, sans doute, la métonymie du nom de l'inventeur. Est-il besoin de dire que pour voir comment ce type opère sur le réel, une définition qui soit opératoire est nécessaire comme pour tous les concepts quels qu'ils soient. La connaissance du fonctionnement de la métonymie permet d'expliquer la polysémie en terminologie. Celle-ci est à exclure, en principe, d'une terminologie. En fait, son existence est due, dans la majorité des cas, aux transferts référentiels par métonymie. Le fait qu'un terme polysémique dans une langue donnée prenne les mêmes valeurs dans d'autres langues ne serait pas autrement explicable. Et les exemples de ce type abondent dans notre corpus français, anglais et arabe.

Il est entendu qu'en terminologie et en documentation, ce phénomène ne doit pas exister et que les termes qui désignent plusieurs concepts doivent être considérés comme des homonymes. C'est le cas, certes, pour certains termes, mais la polysémie en terminologie traduit un lien référentiel qui existe entre deux concepts appartenant à une même classe d'objets: le terme faisceau électromagnétique, par exemple, désigne deux concepts différents en radiocommunication, comme le montrent les définitions suivantes:

\section{Faisceau électromagnétique $\Sigma 1$}

1. Définition: «Ensemble des rayons électromagnétiques issus d'une même source et continus à l'intérieur d'un cône déterminé ou d'un lobe de rayonnement.»

Faisceau électromagnétique $\Sigma^{2}$

2. Définition: «Région de l'espace contenant l'ensemble de ces rayons électromagnétiques.»

C'est la contiguïté référentielle de ces deux objets qui se manifeste dans leurs dénominations par le fait qu'ils ont la même étiquette. Le recours à l'homonymie dans ce cas ne serait pas une solution et risquerait de gommer les relations référentielles qui existent entre les concepts d'un domaine donné.

Mais la solution de la métonymie, qui, donc, se manifeste souvent dans les langues techniques à travers les termes polysémiques, est essentiellement fondée sur une bonne définition du concept. Et c'est pour cette raison que les documentalistes devraient en tenir compte pour éviter les mauvais embranchements dans une hiérarchie.

Un autre exemple de polysémie due à la fois au transfert référentiel que constitue la métonymie et à la variation de facettes ${ }^{13}$ est donnée par le terme appel, qui désigne deux concepts différents mais bien liés. Nous avons constaté en effet à partir des définitions de ce terme un lien référentiel entre les deux concepts qu'il désigne:

\section{Appel $\Sigma^{1}$}

1. Définition: «Une seule communication téléphonique continue.» Synonymes : conversation, appel ayant abouti. Sous-domaine : télétrafic.

Appel $\Sigma^{2}$

2. Définition: «Dans un système automatique, manœuvre effectuee par un demandeur pour se mettre en communication avec l'équipement terminal désiré et, par extension, ensemble des opérations commandées par cette manœuvre.» Sous-domaine: commutation et signalisation téléphonique. 
Le terme appel désigne le début du processus de communication (connexion des équipements) $\Sigma^{2}$ et l'aboutissement de ce processus (conversation) $\Sigma 1$.

Le terme appel $\Sigma^{2}$ est créé par métonymie synecdochique, ainsi que le révèle le rapport de contiguïté référentielle entre les deux processus : le début de l'action (appel $\Sigma^{2}$ ) et son aboutissement (appel $\Sigma 1$ ), c'est-à-dire la conversation ou la réussite de cette manœuvre. C'est dire qu'on prend une partie pour le tout, déplacement de référence qu'on appelle communément «synecdoque de la partie ${ }^{14} \gg$ et qui représente ici une relation partitive.

L'hypallage, que nous avons déjà évoquée dans la deuxième partie, est un facteur commun aux problèmes de variation (la synonymie référentielle ${ }^{12}$ et la polysémie). Elle pose par ailleurs d'autres types de problèmes. Considérons la définition du terme téléphone cellulaire/réseaux de radiocommunication cellulaire (anglais : cellular mobile radio telecommunications system). Définition: «Automobile radio system using lowpower transmitters providing coverage in a limited area but linked with other similar transmitters operating at different frequencies. The whole system enables radio frequencies to be reused economically, and enables good telephone service to be provided in moving automobiles by switching control from one transmitter cell to another as the mobile moves across an area». Ce qui est cellulaire n'est pas la radiocommunication mais la transmission. Dans ce cas, si l'on ne tient pas compte de la définition et de la désignation tropique par hypallage, on risque de classifier ce concept sous la seule facette radiocommunication alors qu'il devrait aussi figurer sous la facette transmission. Un utilisateur d'une base de données ou d'un thésaurus qui s'intéresse uniquement aux systèmes de transmission risque de ne pas trouver ce type particulier, car ce concept se trouverait sous une autre hiérarchie: sous radiocommunication et peut être aussi sous radiotéléphonie.

La métaphore ${ }^{15}$ est aussi fréquente en langue technique. Sa présence pose surtout un problème de reconnaissance automatique. Sa participation à la polysémie à l'intérieur d'un même domaine est très faible. Le seul cas de polysémie lié à la métaphore dans notre corpus est le terme trame, qui désigne deux concepts différents, se classant dans plusieurs facettes, à savoir: Réseaux numériques - Systèmes de transmission, Commutation et signalisation téléphoniques, Transmission télégraphique, Réseaux de communication de données, Radiocommunication. Ce terme représente deux cas de polysémie: polysémie due au transfert sémantique, d'une part, et polysémie due à la variation de facettes, d'autre part. Car dans les quatre premiers sous-domaines, c'est-à-dire les séries $\mathrm{G}, \mathrm{R}, \mathrm{X}, \mathrm{Q}, \mathrm{du}$ CCITT Livre rouge, Termes et définitions, tome X, le concept désigné par trame $\Sigma^{1}$ est grosso modo le même; quelques précisions ou remarques sont ajoutées selon qu'il est envisagé dans telle ou telle série. Mais il reste fondamentalement le même d'après les spécialistes du domaine. Quant au concept désigné par trame $\Sigma^{2}$, il est différent, comme nous le verrons dans les définitions des concepts.

Trame $\Sigma 1$

Définition: «Ensemble répété périodiquement de créneaux temporels consécutifs, dans lequel la position de chaque créneau temporel peut être identifiée.» Sous-domaine: Réseaux numériques - systèmes de transmission, Rec. G, CCITT.

Trame $\Sigma^{2}$

Définition: «Ensemble de lignes réparties à distance égale sur toute la hauteur d'une image et balayées successivement au cours d'un aller et retour du spot analyseur ou synthétiseur dans le sens vertical; une image peut être composée de plusieurs trames.» Sous-domaine : Radiocommunication - télévision, VEI, chap. 60.

\section{CONCLUSION}

La prise en compte de l'ensemble des éléments présentés devrait aider à une meilleure collaboration entre les linguistes et les documentalistes-concepteurs des systèmes 
documentaires. Les termes tropiques poseront lors de la reconnaissance automatique un problème qu'il faudra résoudre. Certaines mesures pourront être déjà prises en ce qui concerne la polysémie liée aux transferts métonymiques et celle liée à la variation de facettes. Dans un cas comme dans l'autre, le moyen le plus sûr d'éviter les confusions lors de l'indexation et lors de l'interrogation des thésaurus serait de proposer un «thésaurus terminologique», idée également formulée par Sagar et Larivière ${ }^{16}$. Il faudrait aussi «appeler» les termes polysémiques nés des transferts référentiels ou de la représentation d'un concept sous plusieurs facettes ou divers angles de la réalité termes multiréférentiels ${ }^{17}$, une appellation qui correspond mieux à la réalité ainsi qu'aux normes terminologiques. S'il n'y a aucun lien entre deux concepts désignés par la même étiquette, c'est qu'il y a indéniablement homonymie.

\section{NOTES}

1. Van Slype définit le langage documentaire comme «tout système de signes qui permet de représenter le contenu de documents dans le but de pouvoir retrouver les documents pertinents, en réponse à des questions portant sur le contenu». Ne sont pas invoqués les critères qu'on utilise en recherche documentaire : auteur du document, langue du texte, date de publication, etc. Les langages d'indexation, p. 19.

2. Le terme langages combinatoires est une autre appellation de langages d'indexation. Ce sont des langages qui permettent de représenter le contenu des documents de manière analytique. La représentation se fait au niveau des concepts relatifs à un domaine donné, soit des descripteurs ou termes dans le titre d'un document, le résumé et le texte du document. Voir Maniez Les langages documentaires et classificatoires : conception, construction et utilisation dans les systèmes documentaires, chapitre 1 .

3. Le thésaurus est un langage contrôlé construit «a priori avant de commencer l'indexation des documents d'une collection et utilisé dans les services de documentation et les banques de données bibliographiques pour l'indexation des documents, c'est-à-dire pour la représentation analytique de leur contentu conceptuel par une série de descripteurs, en vue du stockage et de la recherche de l'information documentaire». Van Slype, Les langages d'indexation, pp. 17-19-22.

4. A. Roman définit la combinatoire comme «la somme des arrangements possibles des phonèmes d'une langue, de ses morphèmes, de ses phonèmes et de ses morphèmes, les combinaisons ainsi produites constituant des séquences ordonnées de longueurs variables: les unités des différents systèmes de cette langue (...). Dans le cadre de la phrase, la combinatoire serait la somme des permutations possibles d'un élément $\mathrm{X}$ d'un ensemble A d'unités de nomination avec les autres éléments de cet ensemble comme il est relié par une relation binaire, de type $<\longrightarrow$ ou de type $\langle-| \longrightarrow$, avec un élément $\mathrm{Y}$ d'un ensemble $\mathrm{B}(\ldots)$.» La création lexicale en arabe, à paraître aux éditions de la Francographie.

5. A. Roman, La création lexicale en arabe.

6. A. Roman, «Les relations syntaxiques», p. 107.

7. «L'hypallage, différemment de la métaphore et de la métonymie, est linguistique et ce n'est, semble-t-il, que par une extension de son emploi qu'elle peut être référentielle. L'hypallage apparaît comme complémentaire de la métaphore et de la métonymie: le versant linguistique de ces deux tropes.» A. Roman, La création lexicale en arabe.

8. «Les Tableaux des Relations» de l'article «ORGANUM» de l'Encyclopcedia Universalis. Les auteurs de cet article proposent une autre manière de mise en relation entre concepts et se démarquent ainsi des classifications de la logique classique. Ils décrivent leur façon de classifier les concepts comme une sorte de «navigation» proposée au lecteur. Nos tableaux disent-ils, ne sont «ni un répertoire organisé des divisions et subdivisions du savoir telles qu'a pu les trouver souhaitables la scolastique des classifications dont reste en fait, imprégné l'ordre positif hérité du XIX ${ }^{e}$ siècle; ni une organisation par degrés hiérarchiques, des notions en quelque mode que ce soit. En un mot, il n'est pas question d'établir pour le lecteur une police d'assurances sur le savoir, c'est-à-dire une image factice, laquelle joindrait, comme en un énorme fagot simulant une armure, les objets de la nature et ceux de la conscience cosmique, scientifique, philosophique du globe» (p. 595).

9. La classification hybride est une classification basée sur des solutions mixtes qui introduisent dans la structure d'un type donné : en partant d'un système monohiérarchique - c'est en tout cas la seule possibilité -on le complète avec quelques facettes auxiliaires. Voir Maniez, Les langages documentaires et classificatoires, pp. 66-67. Pour des exemples, voir W. Mustafa-Elhadi, «La classifications du domaine des télécommunications», La terminologie arabe des télécommunications : fait de variation, chapitre 4.

10. J. Maniez, Les langages documentaires et classificatoires, p. 43.

11. ibid. p. 54 . 
12. Deux termes sont effectivement synonymes si seulement et seulement s'ils désignent le même référent, d'où l'appellation SYNONYMIE RÉFÉRENTIELLE.

13. Ce type de polysémie est relativement plus facile à identifier et à traiter que les autres types. Il s'agit de l'appartenance d'un concept donné à plus d'un sous-domaine. Nous avons adopté certains critères pour identifier ce type de polysémie :

- la référence dans notre corpus d'un terme à deux sous-domaines:

Le Vocabulaire électronique international indique dans ses divers chapitres les divers sous-domaines auxquels un terme donné appartient. Il est de ce fait l'une de nos meilleures références pour le traitement de polysémie due à la variation de facettes ;

- l'identité des définitions des concepts désignés par un terme :

nous avons adopté ce critère en l'absence de toute indication dans notre corpus source, l'indication étant très variable dans les documents cu CCITT notamment : tantôt sont indiqués tous les domaines auxquels un terme appartient, tantôt est indiqué un seul domaine ;

a l'avis des spécialistes du domaine :

lorsqu'il nous a été difficile de décider de l'identité ou de la non-identité de deux ou trois définitions, nous avons consulté les spécialistes. Il est intéressant de signaler certains cas où nous avons constaté une identité de traits de substance ou propriétés d'un objet référentiel, mais une différence de formulation dans les définitions.

Selon les spécialistes (CNET, UIT), les diverses traductions inexactes d'une définition d'un concept peuvent être à l'origine de la variation du contenu définitionnel.

Dans certains cas, le contenu définitionnel d'un concept varie selon le domaine dans lequel il est envisagé, mais les variations ne sont pas, selon les spécialistes, suffisantes pour établir deux concepts effectivement distincts.

14. Tout en marquant les points communs entre la métonymie au sens strict et la synecdoque de la partie, M. Le Guern précise leur différence: «La différence la plus marquante que nous ayons pu remarquer entre cette synecdoque et la métonymie au sens strict réside dans le fait que l'ellipse qu'il faut supposer pour rendre compte du processus linguistique de déplacement de référence est plus complexe dans le cas de la synecdoque de la partie. Alors que dans les deux cas il y a à la fois modifications de la chaîne parlée et glissement de référence, on peut estimer que le second aspect est nettement plus prédominant dans ce type de synecdoque que dans la métonymie» (Sémantique de métaphore et de la métonymie, p. 30).

15. La métaphore proportionnelle favorise le référent beaucoup plus que les mécanismes linguistiques propres à chaque langue. Elle s'établit sur un transfert référentiel analogique d'un objet du monde à un autre objet du monde : la représentation d'un objet du monde coïncide avec celle d'un objet dans un référentiel donné. Pour nommer ce dernier, on prend la même étiquette que propose une langue donnée pour nommer le premier. Une autre langue adoptera le même processus, et c'est là que la traduction littérale pure et simple devient possible, comme nous l'avons vu à travers des exemples de notre corpus pris dans trois langues. Ce fait permet de mieux comprendre l'objet de la terminologie : l'étiquetage des choses. L'étude des tropes en langue technique dépasse dès lors le simple besoin de remédier à la «disette des mots» et nous amène à constater que si la traduction littérale est impossible là où le vocabulaire général est impliqué, elle est par contre possible entre vocabulaires spécialisés. Au demeurant, la traduction littérale est aussi possible sur de nombreux syntagmes longs qui ne sont parfois qu'un résumé de la définition du concept. Une théorie Iinguistique sur la traduction doit nécessairement prendre en compte ces phénomènes particuliers à la terminologie.

16. Voir L. Larivière et Sagar, «Vers un produit unifié en terminologie et en documentation : le thésaurus terminologique», Meta, 34-3, 1989.

17. Ce terme a été proposé par Michel Le Guern, Université Lumière Lyon 2.

\section{BIBLIOGRAPHIE}

BONHOMME, Marc (1987): Linguistique de la métonymie, Berne, Peter Lang.

CHAUMIER, Jacques (1978): Les langages documentaires, le traitement linguistique de l'information documentaire, Paris, Entreprise d'Édition.

CHAUMIER, Jacques (1986) : Les techniques documentaires, Paris, PUF, «Que sais-je ?», 4e éd.

COMMISSION ÉLECTROTECHNIQUE INTERNATIONALE (1970): Vocabulaire électrotechnique international, chapitre 60 .

DEWEZE, André (1981) : Réseaux sémantiques, thèse de doctorat, Université Claude-Bernard Lyon I.

FREGE, Gottlob (1971): Écrits logiques et philosophiques, traduction et interprétation de Claude Imbert, Paris, Seuil.

GRAHAM, Langley (1986): Telephony's Dictionary, Chicago, Illinois, 2 éd.

LE GUERN, Michel (1972): Sémantique de la métaphore et de la métonymie, Paris, Larousse. 
LE GUERN, Michel (1984): «Les descripteurs d'un système documentaire: essaj de définition», Actes du Colloque Traitement automatique des langues naturelles et systèmes documentaires, BES (G.G.), Fauchere (P.M.), Lagueunière (F.), Condenser Supp. 1, Université de Clermont-Ferrand.

LE GUERN, Michel (1989): «Sur les relations entre terminologie et lexique», Actes du Colloque Les terminologies spécialisées - Approche quantitative et logico-sémantique, Montréal, octobre 1988, Meta, 34-3, pp. 340-343.

LOCKE, John (1972): Essai philosophique concernant l'entendement humain, traduit par Coste, texte établi par Émilienne Naert, Paris, Librairie philosophique J. Vrin.

MANIEZ, Jacques (1987): Les langages documentaires et classificatoires : conception, construction et utilisation dans les systèmes documentaires, Éditions de l'organisation, Paris, $291 \mathrm{p}$.

MUSTAFA-ELHADI, Widad (1989): La terminologie arabe des télécommunications : fait de variation, thèse de doctorat, Université Lumière Lyon 2, 2 vol., mai 1989, 629 p.

PEIRCE, Charles Sanders (1978) : Écrits sur le signe, rassemblés, traduits et commentés par Gérard Deledalle, Paris, Seuil.

RANGANATHAN, Shiyali Ramamrita (1957: Prolegomena to Library Classification, London, édité par «The Library Association».

RANGANATHAN, Shiyali Ramamrita (1959): Elements of Library Classification, based on lectures delivered at the University of Bombay in December 1944 and in the Schools of Librarianship in Great Britain in December 1956, édité par B.I. Palmer, London.

ROMAN, André (1985): "Les relations syntaxiques», Cercle linguistique d'Aix-en-Provence, travaux 3 , Aix-en-Provence.

ROMAN, André (sous presse) : La création lexicale en arabe, Éditions de la Francographie.

ROMAN, André (à paraître) : «Les combinatoires fondatrices de l'arabe», Revue de l'École Supérieure Roi Fahd de traduction, Tanger, $25 \mathrm{p}$.

UIT, (Union internationale des télécommunications), CCITT, Livre rouge : Termes et définitions, tome $\mathrm{X}$

VAN SLYPE, Georges (1987): Les langages d'indexation: conception, construction et utilisation dans les systèmes documentaires, Éditions de l'organisation, Paris, $277 \mathrm{p}$.

VIKERY, B. C. (1962): Les techniques modernes de documentation, Paris, Dunod.

VIKERY, B. C. (1963) : La classification, à facettes, Paris, Gauthier-Villars. 